\title{
35 PACIFIC PROVIDER MAKING A DIFFERENCE
}

doi:10.1136/injuryprev-2012-040580d.35

C Timmins*. Auckland Council Injury Prevention, New Zealand

Background Pacific people are overrepresented in injury statistics in New Zealand and injury contributes to significant disparities in health for our Pacific communities.

Waitakere has a long history of working with Pacific communities and providers to raise awareness of injury and build a culture of safety among Pacific peoples.

Aims/Objectives/Purpose To focus on the home environment using a more holistic view of safety across health, injury, housing and immigration, with the understanding that for Pacific families cultural and emotional safety are as vital as physical safety from injury.

Methods Working with our local Pacific health provider using a collaborative framework, positive staff engagement was achieved. Staff continued to participate in the development of a robust home safety checking system which was implemented and reviewed over a 3 year period. An agreed process covering recruitment of families to the programme, a systematic delivery method, options for remediation of issues and a long term review system was developed. Significant professional development of staff was undertaken and a plan for integration of the programme into service delivery was implemented.

Results/Outcomes The final report indicated significant changes in understanding of injury risks, options for remediation and family practice around key risk areas of participating families. This dedicated health provider continues to deliver the home safety checking system as part of their practice.

Significance/Contribution to the Field This project identified a pathway for injury prevention promotion in a key Pacific health agency, providing a continued process to raise awareness and manage risks with pacific families. 


\section{Corrections}

doi:10.1136/injuryprev-2012-

040580d.35corr1

Timmins C. Pacific provider making a difference. Inj Prev 2012;18:Suppl 1 A48 doi:10.1136/injuryprev-2012-040580d.35. An author was left off the author list. The author to be added is Patsi Davies. 